sufficient follow-up to explore the size of these separate effects across childhood. We explored whether birth before full term (gestation 39-41 weeks) and social disadvantage represent a double jeopardy by comparing the size of these separate effects on rates of hospital admission from birth to 10 years.

Methods We linked routine data from birth registration and hospital admissions for all live, singleton births in England in 2005-2006. Children $(n=1,018,136)$ were followed up from discharge from their birth admission to age 10 years, death or study end (31st March 2015). Negative binomial regression models were used to estimate adjusted rate ratios (aRR) with $95 \%$ confidence intervals $(\mathrm{CI})$ for the effect of gestational age $(<32,32-33,34-36,37-38,39-41,42$ weeks) and area deprivation (quintiles) at birth on hospital admissions rates, stratified by age $(<1,1-4,5-10$ years). Population attributable fractions (PAFs) were estimated using the aRR for gestational age and area deprivation. All analysis was conducted in Stata version 14.

Results Hospital admission rates increased strongly with prematurity and area deprivation, even after adjustment for other factors. The effect of gestation was most marked in infancy, when even being born at 'early term' (gestation 3738 weeks) had a stronger effect than living in the most deprived areas $(\mathrm{aRR}=1.37$, 95\% CI $1.35-1.39$ for $37-38$ versus 39-41 weeks; $a R R=1.26$, 95\% CI 1.24-1.28 for the most versus least deprived areas). The effect of gestation persisted though was less marked at age 5-10 years, although even being born 'late preterm' (gestation 34-36 weeks) had a stronger effect than living in the most deprived areas $(\mathrm{aRR}=1.39,95 \%$ CI $1.35-1.43$ for 34-36 versus 39-41 weeks; $a R R=1.29$, 95\% CI 1.26-1.31 for the most versus least deprived areas). In infancy, the PAF for birth before full term $(<39$ weeks) was larger than the PAF for living in the poorest quintile. At age 5-10 years, the PAF for birth before full term was only slightly smaller than the PAF for living in the poorest quintile

Conclusion Gestational age and social deprivation were independent risk factors for hospital admissions throughout childhood. The impact of being born even a few weeks early is of similar magnitude to living in the most deprived areas. Children born early and living in more deprived areas have a double jeopardy.

\section{OP44 BORN TOO SOON: EVIDENCE FROM ONE MILLION CHILDREN ON HOW PREMATURITY AFFECTS HOSPITAL ADMISSIONS IN CHILDHOOD}

${ }^{1} V$ Coathup*, ${ }^{2} E$ Boyle, ${ }^{1} \mathrm{C}$ Carson, ${ }^{2} \mathrm{~S}$ Johnson, ${ }^{1} \mathrm{~J}$ Kurinzcuk, ${ }^{3} \mathrm{~A}$ Macfarlane, ${ }^{4} \mathrm{~S}$ Petrou, ${ }^{1} \mathrm{O}$ Rivero-Arias, ${ }^{1} \mathrm{M}$ Quigley. ${ }^{1}$ Nuffield Department of Population Health, University of Oxford, Oxford, UK; ${ }^{2}$ Department of Health Sciences, University of Leicester, Leicester, UK; ${ }^{3}$ School of Health Sciences, Division of Midwifery and Radiography, City University, London, UK; ${ }^{4}$ Nuffield Department of Primary Care Health Sciences, University of Oxford, Oxford, UK

\subsection{6/jech-2020-SSMabstracts.44}

Background Preterm children are at increased risk of morbidity throughout childhood. However, few studies have investigated the long-term health consequences in relation to the full spectrum of gestational age, week-by-week. This study aimed to estimate the effect of gestational age on hospital admissions from birth up to 10 years of age, explore how rates of hospitalisation change throughout childhood.
Methods Birth registration and hospital admission records were linked for all live, singleton births occurring in England between in 2005 and 2006. Children $(n=1,018,136)$ were followed up from birth admission hospital discharge to age 10, death or study end (31st March 2015). Generalised estimating equations were used to estimate rate ratios (RR) with 95\% confidence intervals (CI). The analysis was repeated looking at infection-related hospital admissions only.

Results Compared to children born at 40 weeks, those born $<28$ weeks had the highest rates of hospital admission throughout childhood $(\mathrm{aRR}=4.89,95 \% \mathrm{CI}=4.55-5.26)$. Even children born at 38 weeks had a higher rate of hospital admission during childhood compared to those born at 40 weeks $(\mathrm{aRR}=1.19,95 \% \mathrm{CI}=1.16-1.22)$. However, the effect of gestational age on hospital admission decreased as the children became older (interaction $\mathrm{p}<0.0001$ ). The most common cause of hospital admissions throughout childhood was infection and similar results were observed when the analysis was restricted to infection-related hospital admissions only e.g. $<28$ vs. 40 weeks, $\mathrm{aRR}=5.57,95 \% \mathrm{CI}=5.01-5.91$. Gestational age had the strongest effect on rates of lower respiratory tract infections, invasive bacterial and viral infection-related admissions.

Conclusion Gestational age is a strong predictor of childhood morbidity, particularly infection-based morbidity, with those born the most preterm at the highest risk. Whilst the effect of gestational age on hospital admission rates decreases with age, an excess risk remained at age 7-10 years.

\section{OP45 WHY DO PEOPLE ATTEND A\&E? A CROSS-SECTIONAL STUDY OF EMERGENCY DEPARTMENTS IN GREATER GLASGOW AND CLYDE}

KA Levin*, S Fleming, E Crighton. Public Health Directorate, NHS Greater Glasgow and Clyde, Glasgow, UK

\subsection{6/jech-2020-SSMabstracts.45}

Background There has been a year-on-year rise in attendance at A\&E departments (EDs) in Greater Glasgow and Clyde (GGC) since 2015/16, despite the opening of alternative units such as medical assessment units and minor injury units (MIUs), and subsequent falling emergency admission rates. This study aims to understand why people choose to attend EDs.

Methods All five EDs in GGC were surveyed over 3 weeks in November/December of 2019. Only those not in crisis were included in the survey. A total of 1196 people were surveyed. Qualitative methods were used to analyse the text data, while chi squared tests compared quantitative data between hospitals. Demographic representativeness was assessed using November/ December 2018 attendance data.

Results Less than a third of survey respondents believed that they had a medical emergency. $42 \%$ were advised to attend by a health practitioner. Twelve per cent attended at A\&E because they could not get a GP appointment with no significant difference between hospitals. Convenience was also cited as a factor and the feeling that this would be the quickest and best place for the person to attend. One third of the four ED respondents tried to get a GP appointment before coming to A\&E, ranging between $28.7 \%$ and $42.2 \%$ 
$(\mathrm{p}=0.008)$. Among the adult patients, approximately a third were given a same day appointment with their GP, and a half were offered an appointment within 48 hours. However, $11.5 \%$ were told they would have to wait more than a week, ranging between 9.6\% and 16.0\% ( $p=0.009)$. 86.3\% of children were given same day appointments. $62.5 \%$ were aware of an alternative service that could treat their urgent health need; almost half cited their GP, yet only $33 \%$ attempted to contact them. MIUs were also cited as an alternative option; over half of respondents across the four EDs attended due to an injury. Only a fifth of respondents sought advice from a health website, while half had a long term health condition.

Conclusion The study indicates that attendance by a proportion of patients could be avoided. The convenience and relatively short wait associated with attendance at $A \& E$ is recognised by the public. Inability to access GPs in a timely manner is an issue, as is the perceived role of GPs. The large proportion of people attending with injuries suggests underutilisation of, or a need for more, MIUs. Health websites should be promoted, especially to those with long term conditions.

\section{OP46 COMPARISON OF SEPSIS RECORDING IN PRIMARY CARE ELECTRONIC HEALTH RECORDS AND LINKED HOSPITAL EPISODES AND MORTALITY DATA: POPULATION-BASED COHORT STUDY IN ENGLAND}

E Rezel-Potts ${ }^{*}$, M Gulliford. School of Population Health and Environmental Sciences, King's College London, London, UK

\subsection{6/jech-2020-SSMabstracts.46}

Background Sepsis is a life-threatening condition resulting from systemic infection. Hospital admissions and recorded deaths for sepsis appear to be increasing nationally, heightening the need for epidemiological studies of sepsis based on accurate and complete data recording across linked records. We aimed to compare the recording of fatal and non-fatal sepsis across primary care electronic health records, hospital episodes and death registrations in England.

Methods A cohort study was conducted including patients registered with the Clinical Practice Research Datalink (CPRD). We analysed linked data for 378 general practices with 1,183,594 patient-years of follow-up and 21,426 first episodes of sepsis from 2002-2017. We searched linked HES admitted patient care records for sepsis events in the 30 days before and after first CPRD diagnosis. We searched the linked ONS death data for patients with sepsis recorded as any cause of death then identified if these patients had sepsis events recorded in the CPRD and HES in the 30 days preceding date of death. We calculated incidence rates and trends in age-standardised incidence in each of the linked records.

Results Among the 21,426 patients with a first episode of sepsis in the CPRD, 4,482 (21\%) had a sepsis event in HES in the 30 days before or after. There were 4,872 patients with sepsis listed as any cause of death in the ONS death data; 2,564 (53\%) had a sepsis event recorded in the CPRD in the 30 days before the ONS date of death compared to $1,187(24 \%)$ in HES. The incidence of new episodes of sepsis was 18.10 per 1000 patient-years $(10.75$ - 28.57) in the CPRD and $7.02(2.83$ - 14.45) in HES.
The mortality rate was 4.12 per 1000 patient-years (1.15 10.41) in the ONS death data. ONS records had peaks in sepsis mortality in 2006 and 2015 which were not reflected in the CPRD and HES records which had similar trends from low to high incidence and steep rates of increase from 2012 to 2017.

Conclusion There was a lack of agreement across data sources for both fatal and non-fatal sepsis events, indicating that relying on singular sources could lead to biased estimates of incidence. Linked electronic health records from primary care, hospital care, and death certificates should be used where possible to increase the accuracy and completeness of epidemiological findings.

\section{Thursday 10 September}

\section{Life Course: Early Life}

\section{OP47 ADVERSE CHILDHOOD EXPERIENCES AND ADULT INFLAMMATION IN THE 1958 BRITISH BIRTH COHORT: COMPARING SINGLE ADVERSITY, CUMULATIVE RISK AND LATENT CLASS APPROACHES}

${ }^{1}$ RE Lacey*, ${ }^{1} S M$ Pinto Pereira, ${ }^{2} \mathrm{~L} L i,{ }^{3,4,5} \mathrm{~A}$ Danese. ${ }^{1}$ Research Department of Epidemiology and Public Health, UCL, London, UK; ${ }^{2}$ Population, Policy and Practice Research and Teaching Department, UCL Great Ormond Street Institute of Child Health, London, UK; ${ }^{3}$ Social, Genetic and Developmental Psychiatry Centre, KCL, London, UK; ${ }^{4}$ Department of Child and Adolescent Psychiatry, KCL, London, UK; ${ }^{5}$ National and Specialist CAMHS Clinic for Trauma, Anxiety and Depression, South London and Maudesley NHS Foundation Trust, London, UK

\subsection{6/jech-2020-SSMabstracts.47}

Background Adverse childhood experiences (ACEs) have been related to poorer health across the life course. Previous studies typically relied on cumulative risk scores or individual adversities measured through retrospective selfreports. However these approaches have important limitations. Cumulative risk scores assume equal weighting of adversities and the single adversity approach ignores the high probability that adversities co-occur. In contrast, latent class analysis (LCA) offers an alternative approach to operationalise ACEs that respects the clustering of adversities and may identify specific patterns of ACEs important for health outcomes. Furthermore, prospective and retrospective reports of ACEs show poor agreement. Therefore, it is important to compare findings based on prospective and retrospective measures in the same individuals. The aim of this study was to compare LCA, single adversity and cumulative risk approaches to operationalising ACEs with inflammation in mid-life, comparing prospectively and retrospectively-reported ACEs data.

Methods Using data on 8,810 members of the 1958 British birth cohort we investigated 12 ACEs - physical, psychological and sexual abuse, physical and emotional neglect, parental mental health problems, witnessing abuse, parental conflict, parental divorce, parental offending, parental substance misuse and parental death. LCA was applied to explore the clustering of prospectively and retrospectively reported ACEs separately. Associations between latent classes, cumulative risk scores and individual adversities with three inflammatory markers (CReactive Protein, fibrinogen and von Willebrand Factor) were tested using linear regression. 